\title{
YBaCuO Josephson generators fabricated by preliminary topology masks
}

\author{
L.S. Revin ${ }^{1,2}$, A.L. Pankratov ${ }^{1,2,3}$, D.V. Masterov ${ }^{1}$, A.E. Parafin ${ }^{1}$, S.A. Pavlov ${ }^{1}$, A.V. Chigi- \\ nev $^{1,2}$, I.V. Rakut ${ }^{2,3}$, A.V. Gordeeva ${ }^{1,2}$, V.O. Zbrozhek ${ }^{2}$, A.V. Blagodatkin ${ }^{1,2}$, L.S. Kuzmin ${ }^{2,4}$ \\ ${ }^{1}$ Institute for Physics of Microstructures of Russian Academy of Sciences, Nizhny Novgorod, Russia, rls@ipmras.ru \\ ${ }^{2}$ Nizhny Novgorod State Technical University n.a. R.E. Alexeev, Nizhny Novgorod, Russia \\ ${ }^{3}$ Lobachevsky Nizhny Novgorod State University, Nizhny Novgorod, Russia \\ ${ }^{4}$ Chalmers University of Technology, Gothenburg, Sweden
}

The investigation of flux-flow regimes in the Josephson junction is of practical interest. In a long junction the mode of fluxon motion may occur under the action of external magnetic field in which solitons are created at one edge of the junction, move along the junction, and are radiated at the other edge. Such regimes can be useful in creation of $\mathrm{THz}$ band oscillators [1], heterodyne and Hilbert spectrometers.

It is well known that the properties of thin YBCO films are very sensitive to the processes of formation of planar structures. New technology of preliminary mask (PM) with $\mathrm{CeO}_{2}$ buffer layer, which determines the necessary topology of the structure directly during the growth of the YBCO film has been developed [24]. The exclusion of the etching operation of the YBCO film from the technological process is important for structures with weak links, where the film is most sensitive to possible destructive factors. The PM method has been successfully used to create Josephson junctions on a bicrystal YSZ substrate with a sublayer of epitaxial cerium dioxide $\mathrm{CeO}_{2}[2,4]$.

As the results of preliminary study, the samples of Josephson junctions with 6-350 $\mu \mathrm{m}$ length and 0.3 $\mu \mathrm{m}$ thickness have been fabricated on symmetric $24^{\circ}$ [001]-tilt bicrystal YSZ substrate.

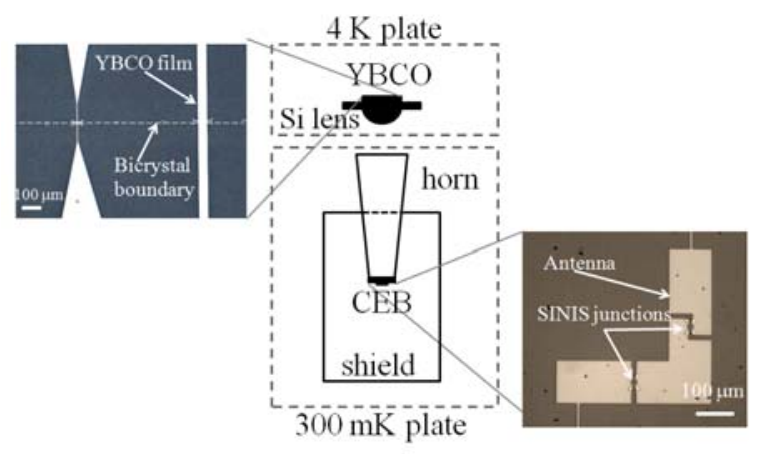

Fig. 1. Schematic of the experiment and samples design.

To register the radiation from long bicrystal junction the sample of Cold Electron Bolometer (CEB) [58] was used. CEBs represent SINIS junctions with nanoabsorber made of aluminum with suppressed superconductivity. One CEB can receive up to $0.5 \mathrm{pW}$ without going into saturation. Based on the requirements of the power load the 2D array of CEB realized as meander-type structure. CEBs are connected in series and parallel at DC for optimal matching with the amplifier. A pair of half lambda dipoles with wide electrodes is connected to another pair by highinductive $1 \mu \mathrm{m}$ wide lines. The design is optimized for the frequency band of 240-280 GHz.
In figure 1 the scheme of experimental setup as well as samples design are presented. The Josephson oscillator chip consists of several junctions integrated with planar dipole antennas. The substrate with the sample was attached to Si hyperhemisphere lens at the $4 \mathrm{~K}$ plate. The bolometer with horn on the back side of silicon substrate was placed at the $300 \mathrm{mK}$ plate. The absorbing shield was used to avoid reflections. The magnetic field perpendicular to the grain boundary of HTSC sample was produced by a current through the copper wire coil, having the inner diameter more than order of magnitude larger than the junction length.

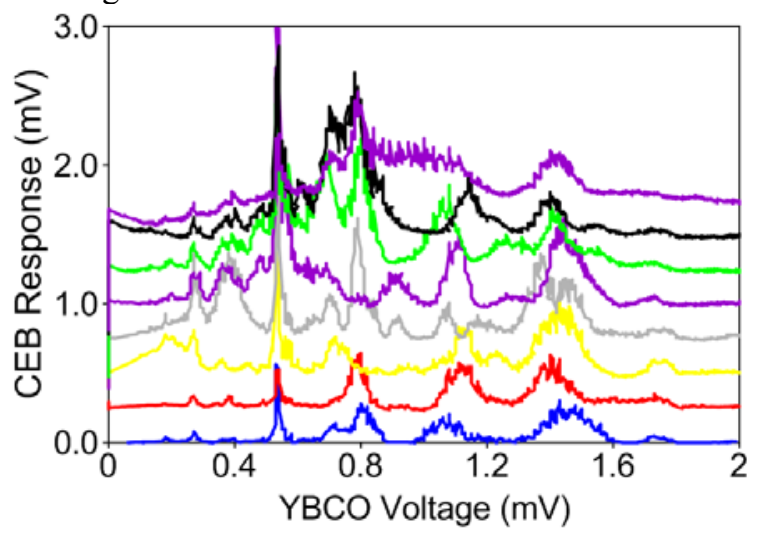

Fig. 2. CEB response for a different magnetic field through the YBCO generator. For clarity, each curve from the second is shifted relative to the other by $0.25 \mathrm{mV}$.

Changing the current through the Josephson junction the bolometer response was measured as well as the voltage on YBCO oscillator (Josephson frequency $\left.f=2 \mathrm{e} V_{\mathrm{YBCO}} / \mathrm{h}\right)$. Figure 2 shows the bolometer response (received power) for different values of the external magnetic field. For clarity each curve from the second one is shifted relative to the other by 0.25 $\mathrm{mV}$. It can be seen that the response represents several distinct peaks and a pedestal. The maximum of this pedestal shifts towards higher voltage with increasing of magnetic field.

Finding the maximum response for each frequency for all values of the magnetic field, we obtain the frequency response of the radiating system together with the receiving system, figure 3. Comparing the result with the CEB amplitude-frequency characteristic based on the calibrated backward-wave source (enlarged part of Fig. 3), it can be concluded that the signal peaks are determined by the characteristic of the receiving system rather than the oscillating one. 


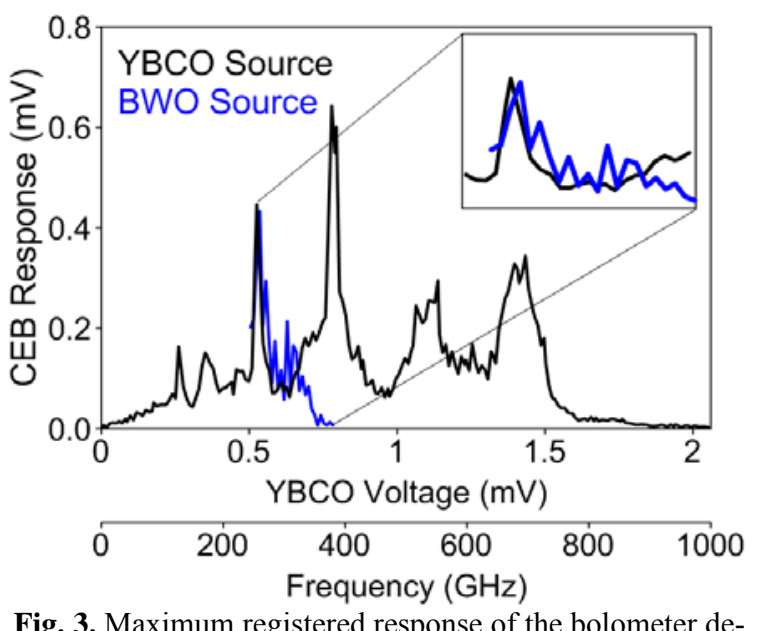

Fig. 3. Maximum registered response of the bolometer depending on the frequency of radiation. Comparison with experiments using BWO.

In conclusion, the samples of YBCO long Josephson junctions on YSZ bicrystal substrate have been fabricated using original technology of preliminary topology mask and good characteristics of the samples have been achieved. The observation of flux-flow regime in long grain-boundary junctions is evidenced. Using the cold-electron bolometer, the subTHz emission up to $900 \mathrm{GHz}$ was registered. Magnetic field controlled regime make long junctions promising candidates for $\mathrm{THz}$ applications. It can also be used as a $\mathrm{THz}$ band cryogenic network analyzer in combination with a cryogenic bolometer.

The work is supported by RSF (projects 16-1910478 and 16-19-10468).
1. Stepantsov, E., Tarasov, M., Kalabukhov, A., Kuzmin, L., Claeson T., THz Josephson properties of grain boundary $\mathrm{YBaCuO}$ junctions on symmetric, tilted bicrystal sapphire substrates // Journal of Appl. Phys. 2004. V. 96, No. 6. P. 3357.

2. Masterov, D.V., Parafin, A.E., Revin, L.S., Chiginev, A.V., Skorokhodov, E.V., Yunin, P.A., Pankratov, A.L., YBCO long Josephson junctions on bicrystal $\mathrm{Zr}_{1-\mathrm{x}} \mathrm{Y}_{\mathrm{x}} \mathrm{O}_{2}$ substrates fabricated by preliminary topology masks // Supercon. Sci. Technol., vol. 30, no. 2, pp. 025007, 2017.

3. Masterov, D.V., Pavlov, S.A., Parafin, A.E., A new approach to formation of the topology of planar structures on the basis of a YBCO high-temperature superconductor // Physics of the Solid State. 2017. vol. 59, pp. 2133-2136.

4. Revin, L.S., Pankratov, A.L., Masterov, D.V., Parafin, A.E., Pavlov, S.A., Chiginev, A.V., Skorokhodov, E.V., Features of Long YBCO Josephson Junctions Fabricated by Preliminary Topology Mask // IEEE Trans. on Appl. Supercon. vol. 28, no. 7, pp. 1100505, 2018.

5. Kuzmin, L., Hoste, S., Ausloos, M., An Array of Cold-Electron Bolometers with SIN Tunnel Junctions and JFET readout for Cosmology Instruments // 8th European Conference on Applied Superconductivity. 2008. vol. 97, pp. 17.

6. Kuzmin, L.S., Devyatov, I.A., Golubev, D., et al. "Cold-electron" bolometer with electronic microrefrigeration and the general noise analysis // Millimeter and Submillimeter Waves Iv. 1998. vol. 34 pp. 193-199.

7. A.V. Gordeeva, V.O. Zbrozhek, A.L. Pankratov, L.S. Revin, V.A. Shamporov, A.A. Gunbina, L.S. Kuzmin, Appl. Phys. Lett., 110, 162603 (2017)

8. L.S. Kuzmin, A.L. Pankratov, A.V. Gordeeva, V.O. Zbrozhek, L.S. Revin, V.A. Shamporov, A.A. Gunbina, S. Masi, P. de Bernardis, "Realization of Cold-Electron Bolometers with Ultimate Sensitivity due to Strong Electron Self-Cooling", Proceedings of 16th International Superconductive Electronics Conference (ISEC'2017), DOI: 10.1109/ISEC.2017.8314194

\section{References}

